\title{
VIRTUAL RESEARCH ENVIRONMENT NANOHLIT FOR HYBRID NANOSTRUCTURES RESEARCH ON THE HYBRILIT PLATFORM
}

\author{
Yu.A. Butenko ${ }^{1, a}$, A.V. Nechaevskiy ${ }^{1}$, I.R. Rahmonov ${ }^{2}$, O.I. Streltsova ${ }^{1}$ \\ ${ }^{I}$ Meshcheryakov Laboratory of Information Technologies of Joint Institute for Nuclear \\ Research, 6 Joliot-Curie St, Dubna, 141980, Russia \\ ${ }^{2}$ Bogoliubov Laboratory of Theoretical Physics of Joint Institute for Nuclear Research, 6 \\ Joliot-Curie St, Dubna, 141980, Russia \\ E-mail: ${ }^{a}$ ybutenko@jinr.ru
}

The paper describes the main components of the NANOHLIT virtual research environment (VRE), developed as part of the joint MLIT and BLTP JINR project, for physical processes modeling in complex systems based on Josephson Junctions. VRE is developed and deployed on the basis of the heterogeneous HybriLIT computing platform (LIT JINR). One of the features of the developed VRE is the use of high-performance calculations on heterogeneous computing platforms. The main goals of creating NANOHLIT were to create a convenient research environment for working with models, performing calculations by various groups of researchers and ensuring storage of simulation results. VRE combines convenient web-based tools for creating models, an interface for performing calculations on the heterogeneous computing platform HybriLIT and tools for results visualization and analysis.

Keywords: information systems, virtual research environment, HPC, software, data storage, mathematical modeling, high-performance computing

Yuri Butenko, Andrey Nechaevskiy, Ilhom Rahmonv, Oksana Streltsova

Copyright $(C) 2021$ for this paper by its authors. Use permitted under Creative Commons License Attribution 4.0 International (CC BY 4.0). 


\section{Introduction}

In hybrid Josephson nanostructures consisting of superconductors and magnetic systems $[1,2]$ an anomalous Josephson effect is observed, which consists in the occurrence of a phase shift in their current-phase relation. This phase shift is proportional to the component of the magnetic moment of the magnetic system, which makes it possible to control the magnetic properties by means of a superconducting current and by superconducting properties by means of magnetic dynamics. Such Josephson nanostructures are promising objects of superconducting electronics and spintronics, which are intensively developing areas of condensed matter physics. Examples of Josephson nanostructures consisting of superconductors and magnetic systems can be a Josephson junction with a ferromagnetic barrier [3] or a system consisting of a Josephson junction and a nanomagnet [4]. One of the important issues in this area is the study of the reversal of the magnetic moment under the influence of an external signal.

Such kind of researches by using mathematical modeling methods need both resourceintensive calculations and calculations for a large number of model parameter values. So use of highperformance computing platforms on which calculations can be organized with both parallel programming technologies and data parallelization technologies are necessary. An important part of the research effective process is a convenient tool for visualization and analysis of modeling results.

The above factors, as well as the need for the research results exchanging by various scientific groups, led to the creation of a virtual research environment NANOHLIT, as a joint project of a group of researchers from the BLTP and the MLIT of the Joint Institute for Nuclear Research. VRE NANOHLIT based on the heterogeneous computing platform HybriLIT [5], which provides both the possibility of developing an IT project and resources for parallel calculations [6,7], a system for storing models and data, as well as resources for VRE deployment and support.

It should be noted that the term virtual research environment (VRE) is usually used to refer to developed digital environments that are designed to meet a number of needs of the research community [8]. VRE means an environment with a set of tools and technologies necessary for scientists to conduct their research, interact with other groups of scientists, use resources and technical and computing infrastructures [9]. The functionality of the developed VRE NANOHLIT includes such capabilities as creating models, performing calculations, including on hybrid computing architectures, tools for data analysis, combined on the basis of web technologies into a single software and information environment with support for the collective work of research groups. At the moment, users could make a research with available systems of superconductor/ferromagnetic/superconductor [10], allowing for the calculation of time dynamics and parametric dependence, and system consisting of a long Josephson junction and a nanomagnet. Also, the developed environment is planned for teaching students to work with models of systems built on Josephson junctions, which requires separate consideration when developing the system.

\section{VRE backend description}

For modeling physical processes in complex systems based on Josephson junctions VRE uses massive parallel processing and high-performance computing based on HybriLIT platform.

As noted above, a key feature of the developing NANOHLIT research environment is the need to provide convenient and secure access to high performance computing resources (CPU, graphics processors, and data storage) for various research groups. A typical HPC platform user process: model building, design and implementation of a computing scheme is carried out on a user's resources, then data is transferred to the HPC platform on which the applications are launched, and the results of the simulation are copied back to the user, where they are analysed and visualized using different tools.

In this case, it becomes actual to develop a special web-based environment for modelling in a single information space, as well as providing an opportunity for different research groups to organize 
joint research, exchange models and calculations results. Developed web-service allows user to interact with the resources of the computing platform: run models with different parameters, receive the status of its execution and the results. The implementation of such a function is possible using the client-server architecture of the web service, in which the client part provides the user interface, and the server part implements interaction with the HybriLIT platform. The scheme and the description of the service architecture are described in [11].

The service are implemented as a web application and has a client-server architecture. The servers are Node.js, and the clients written using the Vue.js library. The service provide convenient and safe access to the HybriLIT platform and the ML/DL ecosystem, which comprises a large number of frameworks and tools. This approach has the following advantages:

- the service provides authorized access to the computing components of the HybriLIT platform using the SLURM batch system;

- on the basis of web technologies, a common interface for researchers is implemented for interacting with the MariaDB database and file storages;

- the service provides a user interface for storing, modifying and adding data.

In addition, all calculations are performed on a remote dedicated server, which does not require computing resources from the user's device. The server-side (backend) is the software and hardware part of the service, which implements application programming interfaces (APIs) for interaction of the client-side (frontend) with the service components without directly accessing them from the frontend using POST requests. It simplifies work with the platform for a group of researchers and isolates different use cases depending on access rights. The core of the backend is the Node.js technology. This software platform allows one to implement a fast, asynchronous, eventdriven API server. Node.js also adds the ability for JavaScript to interact with $\mathrm{I} / \mathrm{O}$ devices via its programming interface and to connect other external libraries, which are written in different programming languages (C/C++, Python, etc.).

\section{VRE frontend}

At the moment VRE NANOHLIT users are divided into three roles: "administrator", "researcher" and "student" with different levels of access to the system shown in the diagram below (Fig. 1). The block that is numbered in the diagram with "1" is responsible for filling the system with models (Model Bank). This block is available only for "administrator" and "researcher", the difference between "administrator" and "researcher" rights is the ability to configure access rights and manage the contents of the "Model Bank".

The second block (indicated by the number "2") implements the workflow of all users of the system. It allows one to create own models based on the models presented in the "Model Bank", edit model (modify model elements and change parameter values), perform calculations on selected platforms with certain sets of parameters and view the results of calculations. The third block (number "3"), allows the evaluation of the calculations performed by the student, the block is available for the "administrator" and "researcher". 


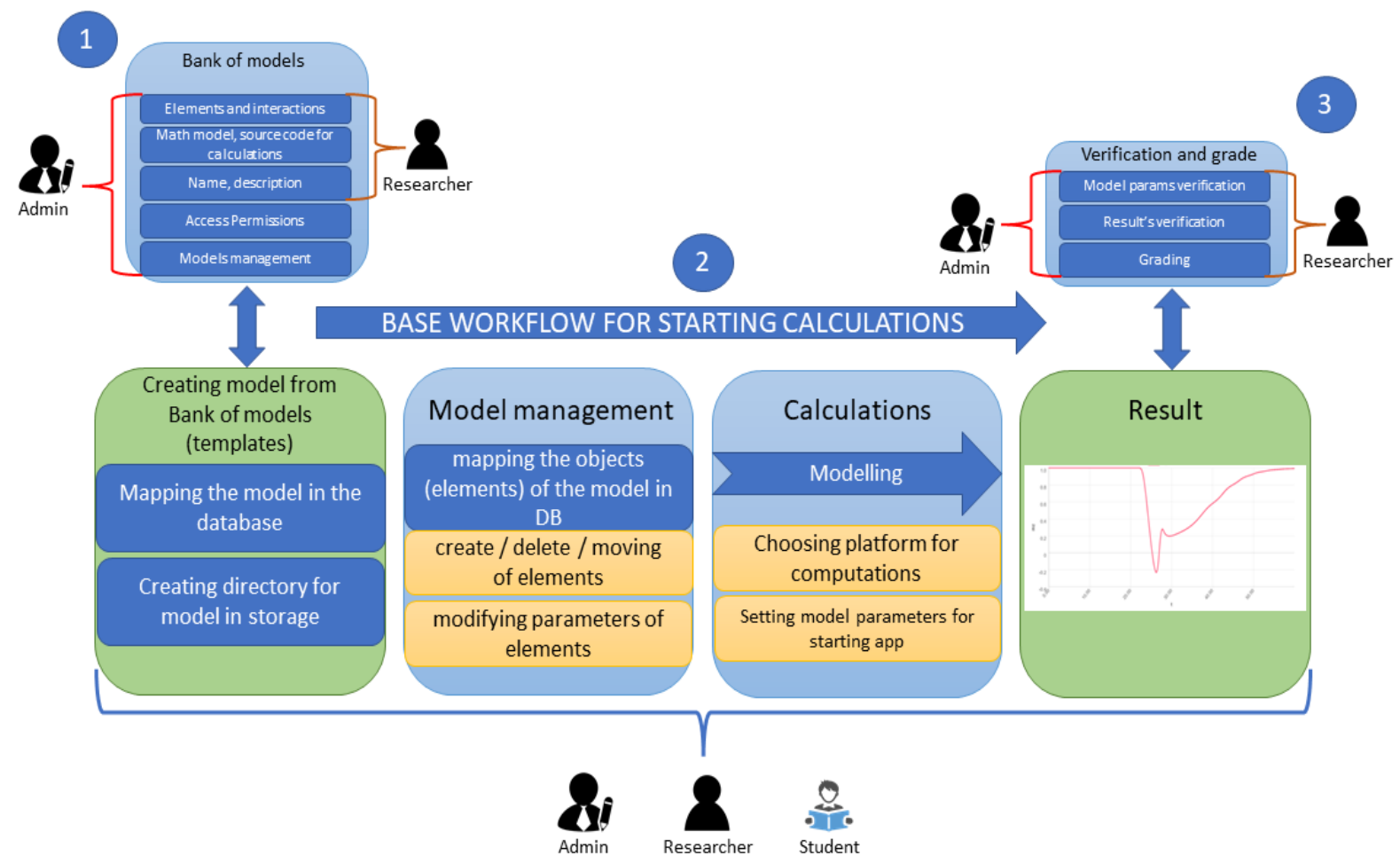

Figure 1. Roles and base workflow

Let's describe the user's interaction with the system in more detail. First of all, in order to interact with the platform, the user needs to have an account on the HybriLIT platform. At the beginning of the work, the user selects the necessary object from the "Model Bank". Based on the selected model, a new object is formed for conducting research (point 2, Fig.2). After that, a form for constructing a computational experiment becomes available to the user. For this purpose, a module has been implemented for the formation of mathematical models, input of initial and boundary conditions, parameters and control functions for the systems under study, depending on the selected algorithm (point 3, Fig.2). After correctly filling in all the necessary parameters, you can run the task on the account by pressing the "RUN" button. The list of elements for the model configuration is implemented in the form of JSON objects stored in the database. For example, the structure of the superconductor/ferromagnet/superconductor (SFS) object includes: identifier, name, direct position on the diagram, location of the script to run in the SLURM batch system, parameters for calculation. Depending on the user's choice, the corresponding algorithms are launched to perform calculations on the HybriLIT platform. As a result, the task ID will come from the server as a response (item 4, Fig.2). For the convenience of the user, the ability to view data from files with results in the form of graphs via the web interface is implemented. To build a graph, the user selects a file from the list and the type of graph (p.5,6, Fig.2). In addition, it is possible to combine data from different files on the same chart. 


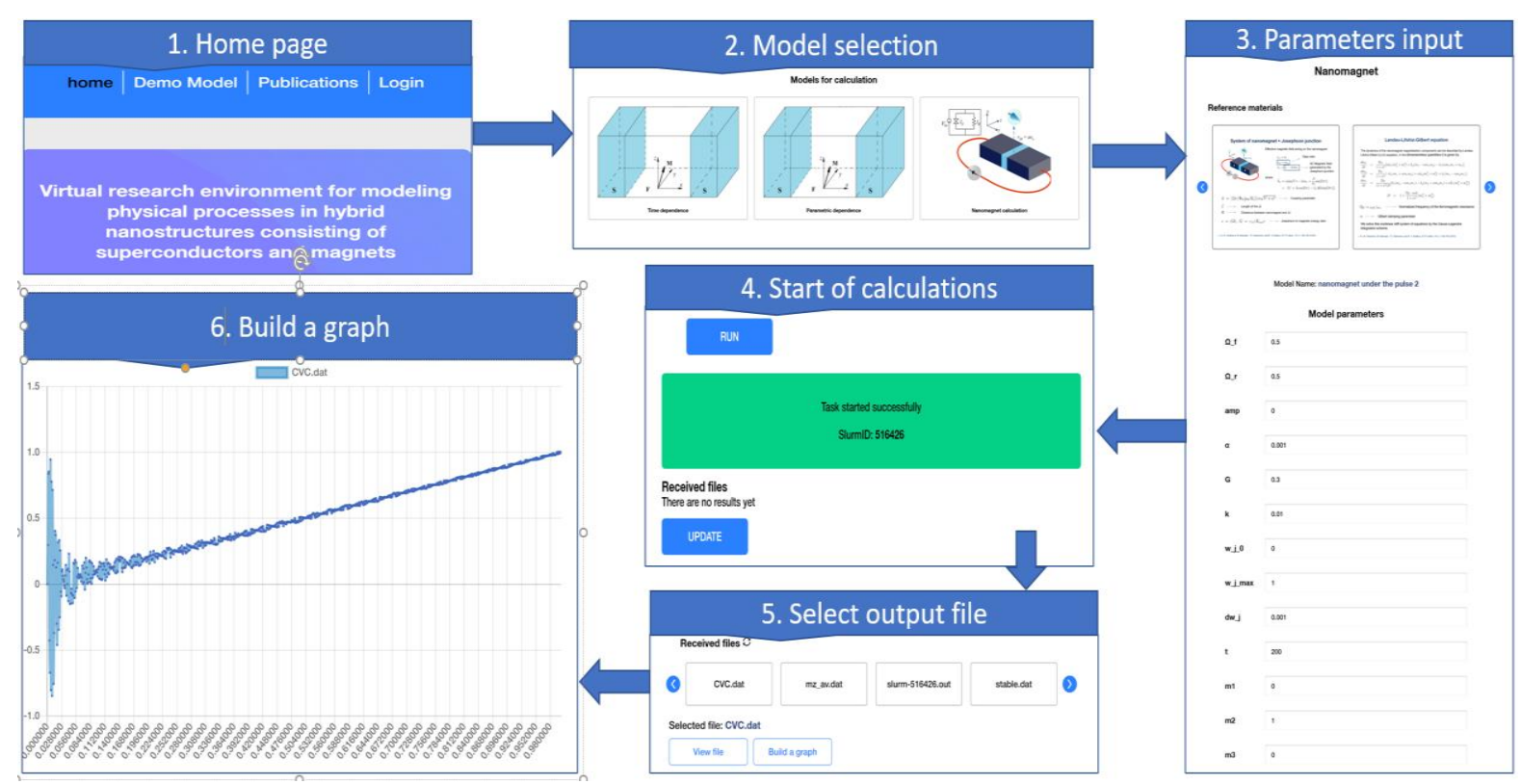

Figure 2. Scheme of user interaction with the system web interface

\section{Conclusion}

To solve the tasks of mathematical modeling of physical phenomena in hybrid nanostructures comprising superconductors and magnets, a prototype of the virtual research environment (VRE) was developed. The developed VRE prototype allows one to create computing experiment from a given set of models online. The implemented algorithms and tools allow one to perform a parallel calculations on HybriLIT platform, as well as analyze results. The research environment is actively developing, supplemented with new relevant models and tools.

It is planned to use the VRE NANOHLIT into the educational process for research Josephson junctions systems by mathematical modeling for students at the JINR UNC, Dubna State University and other universities.

The developed approaches and IT solutions can be used to create virtual research environments for solving applied tasks in different fields of science.

\section{Acknowledgement}

The reported study was funded by RFBR according to the research project № 20-37-70056.

\section{References}

[1] J. Linder and W. A. Jason, Nat. Phys. 11, 307 (2015).

[2] S. Mai, E. Kandelaki, A. F. Volkov, and K. B. Efetov, Phys. Rev. B 84, 144519 (2011).

[3] F. Konschelle and A. Buzdin, Phys. Rev. Lett. 102, 017001 (2009)

[4] Cai and E. M. Chudnovsky, Phys. Rev. B 82, 104429 (2010).

[5] Gh Adam., et al. IT-ecosystem of the HybriLIT heterogeneous platform for high performance computing and training of IT-specialists // Selected Papers of the 8th International Conference «Distributed Computing and Grid-technologies in Science and Education»(GRID 2018), Dubna, Russia, September 10-14, 2018, CEUR-WS.org/Vol. 2267 
[6] Balashov N.A., et al. Service for parallel applications based on JINR cloud and HybriLIT resources // 23rd International conference on computing in high energy and nuclear physics (CHEP 2018), EPJ Web of Conferences, 2019, 214(07012), DOI:10.1051/epjconf/201921407012

[7] Butenko Yu.A., Marov D.M., Nechaevskiy A.V., Podgainy D.V. Development of a service for conducting radiobiological studies on the HybriLIT platform // CEUR Workshop Proceedings, 2020, 2743 , pp. 26-33

[8] M. Barker, S. Delgado Olabarriaga, N. Wilkins-Diehr, S. Gesing, D.S. Katz, S. Shahand, S.Henwood, T. Glatard, K. Jeffery, B. Corrie, A. Treloar, H. Glaves, L. Wyborn, N.P. Chue Hong, A. Costa The global impact of science gateways, virtual research environments and virtual laboratories. // Future Generation Computer Systems 95 (2019) 240-248

[9] Jisc, Virtual research environment programme, 2014, URL: http://webarchive.nationalarchives.gov.uk/20140702163345/http://www.jisc.ac.uk/whatwedo/program mes/vre.aspx. (Accessed: 20 May 2018).

[10] P.Kh. Atanasova, S.A. Panayotova, I.R. Rahmonov, Yu.M. Shukrinov, E.V. Zemlyanaya, M.V. Bashashin Periodicity in the Appearance of Intervals of the Reversal of the Magnetic Moment of a $\varphi 0$ Josephson Junction. JETP Letters. 2019; 110(11):722-726. (In Eng.) DOI: https://doi.org/10.1134/S0021364019230073

[11] Yu.A. Butenko, D.M. Marov, A.V. Nechaevskiy, O.I. Streltsova, I.R.Rahmonov, M.V. Bashashin Development of a Virtual Research Environment for Modeling Physical Processes on the HybriLIT Platform in Systems Based on Josephson Junctions // ModernInformationTechnologiesand ITEducation. Vol. 16, No. 3. 2020 DOI: https://doi.org/10.25559/SITITO.16.202003.633-642 